IWSCFF 17-08

\title{
IN-ORBIT EXPERIENCE AND LESSONS LEARNED FROM THE AVANTI EXPERIMENT
}

\author{
G. Gaias* and J.-S. Ardaens ${ }^{\dagger}$
}

\begin{abstract}
This work addresses results and practical challenges of the AVANTI in-flight demonstration. This endeavor realized a fully autonomous rendezvous to a noncooperative target in low Earth orbit, in the separation ranges between tens of kilometers to 50 meters. Lessons learned gained from the flight campaign are reshaped as design guidelines for handing over the peculiar guidance navigation and control system - AVANTI-concept - to future rendezvous missions.
\end{abstract}

\section{INTRODUCTION}

The AVANTI (Autonomous Vision Approach Navigation and Target Identification) experiment recently demonstrated the viability of a purely vision-based approach to autonomously rendezvous a passive target in low Earth orbit (LEO). ${ }^{1}$ Within such in-flight demonstration, in fact, the Earthobservation small satellite BIROS has been used to chase the BEESAT-4 CubeSat, from far-range down to circa $50 \mathrm{~m}$ of inter-satellite distance, in a fully autonomous fashion. ${ }^{2}$

Compared to other multi-satellite missions flown so far in LEO (e.g., Orbital Express, ${ }^{3}$ PRISMA $^{4}$ ), the main peculiarity of the AVANTI demonstration was the incontrovertible noncooperative mission scenario. As a matter of fact, the images taken by BIROS constituted the unique source of observations available in real-time to perform the relative navigation task. This was due to the absence of any form of communication between BIROS and the target body and to the lack of external navigation sources usable a/o accurate enough for inter-satellite distances below few kilometers.

To meet its ambitious goals, AVANTI pursued a low-cost minimalistic design approach with no impact on the design of the chasing spacecraft: BIROS already featured a propulsion system and a star-tracker sensor. This latter has been used as far-range camera and no further formation-flying specific sensors and actuators have been embarked on the already designed BIROS satellite. In addition, AVANTI exploited the opportunity that the BIROS spacecraft embarked a single picosatellite launcher device to release in orbit the BEESAT-4 one-unit CubeSat of the Technical University of Berlin. ${ }^{5}$ So far pico/nano satellites have been usually deployed from the upper stage of a launch vehicle or from the international space station with the goal of getting as far as possible from them to reduce the collision risk. In this case, instead, the BEESAT-4 ejection has been considered as an appealing opportunity to generate in a cheap way a target to support proximity operations activities. This aspect embodies a further innovation brought by AVANTI and the resulting separation strategy design has been addressed in References 6 and 7, leading to the well-known in-flight events. ${ }^{2}$

\footnotetext{
* Research Engineer, GSOC/Space Flight Technology, Münchener Str. 20, 82234 Wessling, Germany.

${ }^{\dagger}$ Research Engineer, GSOC/Space Flight Technology, Münchener Str. 20, 82234 Wessling, Germany.
} 
Originally planned to start immediately after the release of BEESAT-4, the AVANTI experiment could only take place two months after it, since due to some scheduling conflicts in the mission timeline the experiment commissioning could not be performed before the BEESAT-4 deployment deadline. The latest possible time-limit to eject the picosatellite, in fact, was a hard constraint driven by the endurance of its battery, which had been recharged for the last time before the satellite integration at the launchpad. As a matter of fact, postponing the experiment commissioning phase already with the target satellite free-flying in space turned out to be an extremely valuable situation. From the one hand, it extended the flight-time allocated to AVANTI. From the other hand, AVANTI became a unique testbed to stepwise familiarize with the vision-based approach, with increasing levels of complexity and autonomy.

Indeed the vision-based approach demonstrated by AVANTI is very appealing for future on-orbit servicing and debris removal missions: simply using a passive monocular camera has no impact on the spacecraft system design but it allows to safely carry out the first phase of the rendezvous. AVANTI itself is an example of the high level of portability of such guidance navigation and control (GNC) concept: de facto its spaceborne GNC system has been integrated into a satellite not specifically designed to support formation-flying activities. At the same time, AVANTI has been an extremely realistic technological demonstration for future LEO applications, since it took place on a general orbit scenario, strongly perturbed by differential aerodynamic drag and presenting eclipses that lead to periodic outages of the visibility of the target satellite. This paper sheds light on some practical aspects encountered during the course of the flight activities. The experience gained turns into lessons learned to exploit the AVANTI-concept in possible future missions.

\section{IN-ORBIT EXPERIENCE}

AVANTI was one of the secondary scientific experiments to be accomplished within the FireBird mission. ${ }^{8}$ This is a small-scale scientific mission of the German Aerospace Center (DLR) for Earth observation and hot spot detection comprising a loose constellation of two satellites: TET-1, ${ }^{9}$ already launched in July 2012, and the Bi-Spectral InfraRed Optical System (BIROS), launched on the $22^{\text {nd }}$ of June 2016. BIROS has been injected into an almost circular, Sun-synchronous local time of ascending node 21:30, $515 \mathrm{~km}$ high orbit. Afterwards, on the $9^{\text {th }}$ of September 2016, BIROS released BEESAT-4 in-orbit by means of a single picosatellite launcher device which provided an equivalent separation delta- $v$ of circa $1.5 \mathrm{~m} / \mathrm{s} .{ }^{6,7,2}$ While carrying out its independent experimental activities, BEESAT-4 has been used as noncooperative target for the sake of the AVANTI demonstration.

The timeline of the whole in-orbit experience collected to prepare and support AVANTI is shown in Figure 1 against the explored inter-satellite ranges. The experiment commissioning phase began shortly after the ejection of BEESAT-4, in parallel to the completion of the BIROS bus validation. Its overall duration occupied the majority of the flight-time since it comprised the stepwise verification of all the interfaces and functionalities required to support autonomous formationflying activities. Particularly, the AVANTI GNC system made use of the following essential capabilities of the BIROS platform: attitude determination and control, absolute orbit determination, power/thermal/communication management, and activation of the propulsion system. From the AVANTI GNC side, instead, the following functionalities have been verified: the core relative GNC and safety monitoring tasks (e.g., interfaces with the star-tracker and behavior of the flight $\mathrm{SW}$ ), the attitude guidance function (e.g., selection of the best-suited attitude mode in compliance with the autonomous GNC activities), and experiment data handling (e.g., pictures and data storage and 
down-link). At the same time, the preliminary phase has been also used to verify the experiment ground-segment, that is all the specific tools required for monitoring and supporting this technology demonstration (e.g., post-processing relative precise orbit determination facility).

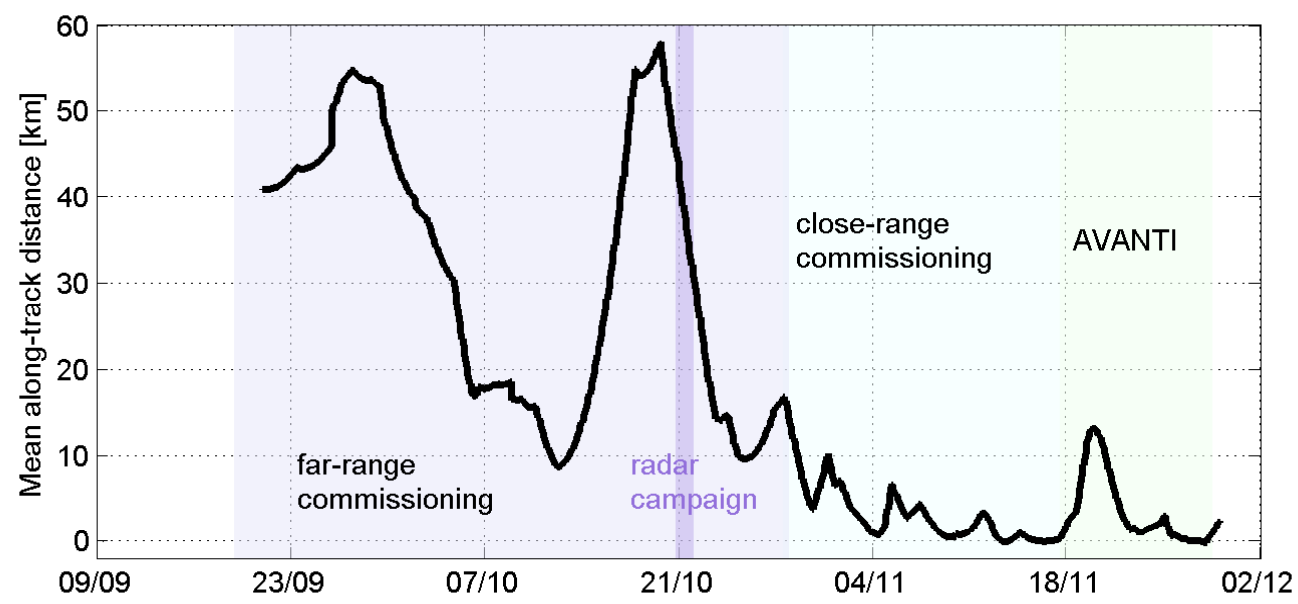

Figure 1: In-orbit phases for the preparation and execution of AVANTI.

In parallel to these functional verifications, the experiment commissioning phase was meant to investigate several aspects of the visual-based angles-only navigation, critically exposed in References 10 (i.e., ground-based re-processing) and 11 (i.e., performance of the onboard navigation system). To this end, the first phase of the commissioning focused on the far-range domain, whereas the second part has been used to investigate the mid- to close-range regions. At far-range the main difficulties lay in the ability to distinguish the target and to perform a meaningful orbit determination given the hardly observable variations of relative motion at such distance. As independent verification of the line-of-sight relative navigation results a radar campaign has been conducted on the 20-21 October with the support of the German Tracking \& Imaging Radar facility. Such radar observations have been used to perform radar-based picosatellite absolute orbit determination, thus obtaining a relative reference solution, with respect to the BIROS GPS-based absolute orbit. Results revealed to be consistent, achieving the same accuracy at least for the two lateral components. ${ }^{10}$ The first two weeks of November, instead, have been dedicated to collect experience in imaging BEESAT-4 at closer distances, traveling through the mid-range domain (i.e., from $10 \mathrm{~km}$ to few hundreds of meters), and reaching two times a relative distance below $200 \mathrm{~m}$. At close-range, the main challenges are related to the fact that the target starts appearing very bright and large in the pictures, and the differential aerodynamic drag perturbation drastically changes, due to the tracking observation attitude profile that is required to keep BEESAT- 4 in the camera field of view.

Once completed the aforementioned preparatory phases, in the second half of November, the fully autonomous activities could begin and the AVANTI experiment could be successfully carried out. Reference 1 presents the guidance, navigation, and control flight results achieved during such autonomous activities. 


\section{GNC ARCHITECTURE ASPECTS}

The first lesson learned from AVANTI corresponds to the main achievement of such demonstration: a purely angles-only (AO) navigation approach is feasible and safe despite navigation uncertainties and maneuver execution errors, even in the challenging environment of targeting a noncooperative object in LEO. As mentioned in the introduction, the major benefit of exploiting solely a monocular camera is the minimal impact on the chaser spacecraft design. Nevertheless, this comes at the cost of solving the weakly observable problem of reconstructing the relative state out of a sequence of bearings-only observations. Basically, complexity moves from the spacecraft design (i.e., sensors, mass/power, thus costs) to the algorithms of the GNC system.

The key of success of AVANTI is to be found in the peculiar design of the GNC system, customized to cope with the intrinsic drawbacks of an AO approach: a passively safe guidance profile is generated to compensate navigation and, consequently, control performance anisotropy. Out of the relative orbit determination, in fact, the achievable longitudinal accuracy (i.e., corresponding to the along-track direction at far-range) is way much worse than the lateral accuracy (i.e., perpendicular to the line-of-sight). At the same time, the overall navigation accuracy remarkably improves when the distance between the satellite decreases. In such a situation, a collision-free approach can be achieved exploiting a smoothly-drifting transfer trajectory that presents (anti-)parallel relative eccentricity and inclination vectors and that shrinks its size in the plane perpendicular to the orbit velocity to reduce the overall 3D distance to the target. The GNC system designed to support AVANTI realizes - and demonstrated in-flight - such strategy, deserving the appellation of AVANTIconcept. It, in fact, embeds the generation of delta-v optimum passively safe rendezvous trajectory with an on-line independent monitoring of the one-orbit minimum lateral inter-satellite separation, with the authority to preemptively break the rendezvous in case of any contingency (thus exploiting the intrinsic advantage of a passive collision avoidance strategy).

The peculiar design of such AVANTI-concept can be explained with the support of Figure 2. Here, the left side presents a detail of the overall functional view discussed in Reference 1. Highlighted are the main modules, referred as to AVANTI and OSM (onboard safety monitoring), and their interfaces to the BIROS AOCS system. The right view focuses on how the main functions interact with each other: the scheme tries to condense functional relationship with sequential connections.

The linking between navigation system and G\&C determines how the overall control loop is closed. In the AO framework, key point is to stepwise refine both navigation and control solutions, despite a weakly observable navigation. Therefore, it is important to balance the promptness of the control reaction, given the accuracy that is actually achievable and realizable without a useless waste of delta-v. The G\&C is implemented with the typical receding finite-time horizon of the model predictive control (MPC). The prediction horizon equals the time from the plan update moment (i.e., plan generation state) to the aimed final time of the whole rendezvous horizon. Whereas the control horizon is the time to achieve the first incoming intermediate way-point (not shorter than two orbital periods). According to the implemented solution scheme, this requires up to 4 impulsive maneuvers, internally managed as a state machine (more details in Figure 6 of Reference 12). Thus, the control loop is closed at each refinement of the guidance plan.

The originality of the approach is how to actually solve the optimal planning problem. This prescribes the achievement of an aimed relative state at a given future time, in a fuel efficient, safe, and feasible manner, that is in compliance with several operational constraints dictated by satellite bus and experiment needs. The convenient set of variables represented by the relative orbital 

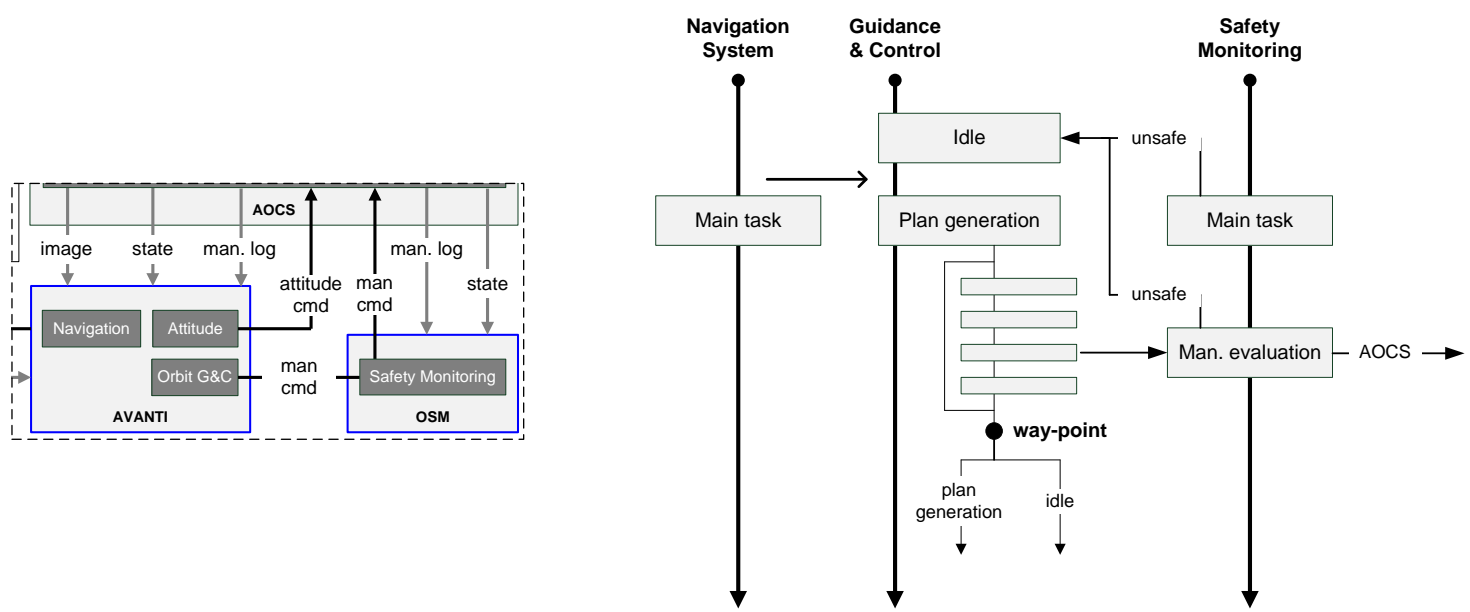

Figure 2: Left: Detail of GNC SW functional view (zoom from Figure 3 of Reference 1). Right: time and logic connections among the main GNC tasks.

elements (ROE) is chosen. These, in fact, allow recasting such time-dependent optimal control problem into a geometrical minimum-path problem in the ROE space. ${ }^{13}$ And the guidance solution is the sequence of way-points, corresponding to passively safe relative orbits, to reach the aimed final orbit. To achieve each intermediate way-point, maneuvers are scheduled in time-constraintfree slots through a locally delta-v optimal analytical burns' scheme (i.e., Eq. (8) of Reference 14 for the out-of-plane correction and the option N12 of Table 2 of Reference 14 for the in-plane reconfiguration). As a result, the implemented architecture exploits typical benefits of MPC like the capability to enforce constraints on input (i.e., time constraints on the time of the maneuvers) and outputs (i.e., end-condition and passive safety), and to optimize a performance index (i.e., fuel consumption). At the same time, it mitigates the MPC drawbacks of requiring a prediction model and a larger computational load of classical (linear) control methods. Regarding the first aspect, the ROE-based model for the perturbed relative motion in near-circular orbits of Reference 15 is used. It presents a simple and compact formulation, though being accurate over extended time periods. The planning problem, on the other hand, is reduced to the solution of a linear convex problem in the ROE state. ${ }^{13}$

The linking between G\&C and safety monitoring, instead, realizes the implementation of the AVANTI safety concept detailed in Reference 12. OSM constantly monitors the safety, in the sense of collision avoidance, of the BEESAT-4-BIROS formation. To realize a robust approach, safety is assessed independently from the onboard navigation solution: the latest best available knowledge of the relative state produced by the ground-based data re-processing is used as reference trajectory and propagated in time. The criterion to assess the safety of the relative trajectory is based on the properties of the uncertainty distribution of the one-orbit minimum radial-normal ( $\mathrm{RN}$ ) distance between the two spacecraft (see Eq. (1) of Reference 12). OSM exercises an onboard preemptive action since it evaluates each maneuver commanded by the AVANTI module and forwards it to the AOCS of BIROS only if the post-maneuver trajectory is considered to remain safe within a prescribed amount of hours following the burn.

As example of the GNC behavior, results from the close-range commissioning phase are presented in Figures 3 to 5. 

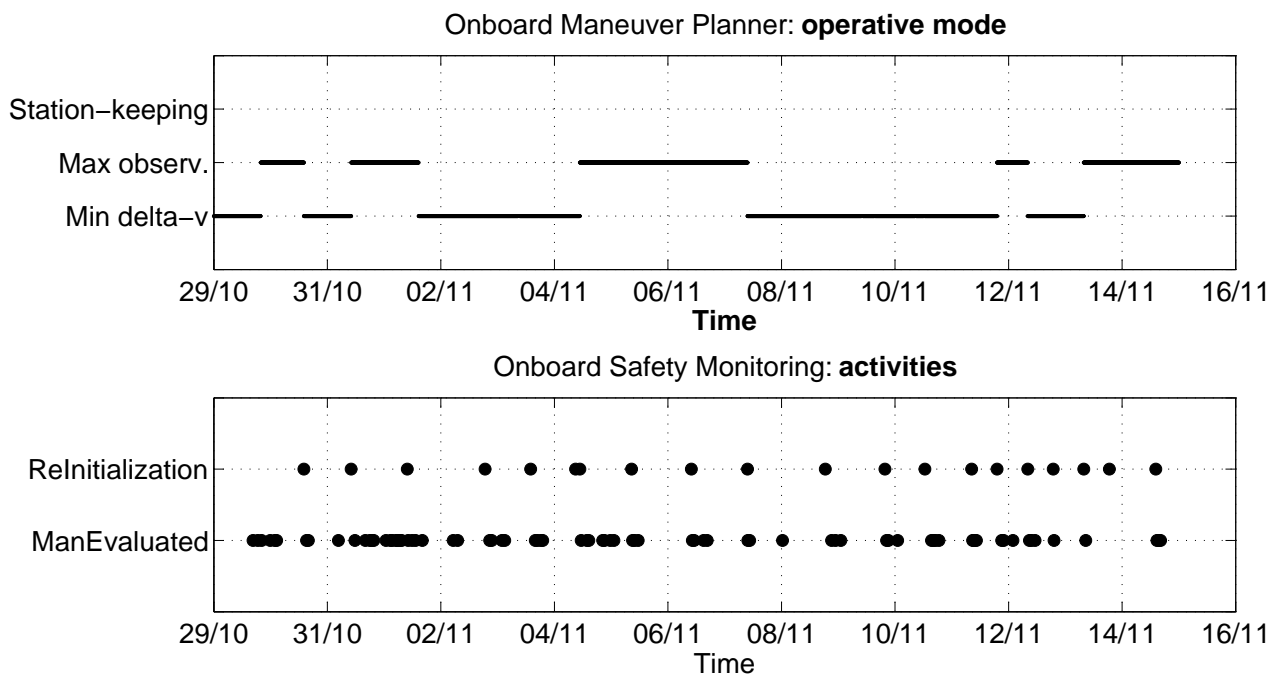

Figure 3: G\&C and OSM interaction during the close-range commissioning phase.

Figure 3 presents how G\&C and OSM cooperated: a certain number of maneuvers have been evaluated and executed, with OSM re-initialized on an average twice per day. The output of the evaluation process is plotted in Figure 4. With lower bound of the one-orbit RN minimum distance it is meant the mean minus 3 times the standard deviation value of its distribution given the uncertainties in the propagation initial condition and accumulated maneuver execution errors. Around the $5^{\text {th }}$ of November such value decreased in correspondence with the reduction of the magnitude of the relative inclination, to get closer to the target. This can be clearly noted observing the relative trajectory that BIROS performed with respect to BEESAT-4 (Figure 5). It presents the well-known AVANTI spiraling profile: more approaches have been carried out with smaller relative orbit size. By referring to the upper plot of Figure 3, one can note that the maneuver planner operated in two different modes during the close-range commissioning phase. This is a further degree of flexibility provided by the AVANTI GNC system and it regards how the optimal planning problem is solved. As explained in Reference 13, the max-observability mode is used to intensify the occurrence of maneuvers, being it related to the number of intermediate way-points that are exploited (i.e., length of the control horizon w.r.t. the prediction one). On the other hand, the minimum delta-v option uses the smallest number of maneuvers strictly needed by the implemented analytical control scheme (i.e., the control horizon is set equal to the prediction one). Structurally this latter option cannot achieve the same overall accuracy performance of the max-observability operative mode. Nevertheless, it presented the practical advantage to reduce the number of thruster activations, being the thruster firing attitude mode conflicting with the optimal orientation of the star-tracker to target. This aspect was particularly interesting in some phases of the close-range commissioning, when the maximization of the collection of visual data was sought.

The commanded delta-vs corresponding to the maneuver evaluations of Figure 3 are plotted in Figure 6. Red diamonds mark maneuvers that failed to occur, due to some communication problems between AOCS and thrusters (shortly after completely solved). The key consideration is that, thanks to the AVANTI-concept, such issues did not pose any danger to the safety of the space segment. As depicted in Figure 2-left, in fact, OSM receives a feed-back from the AOCS system of BIROS and 


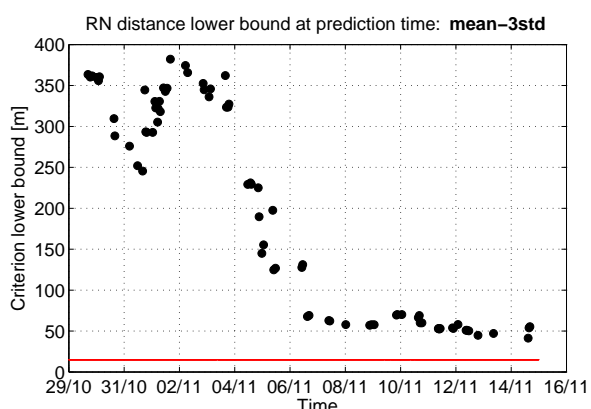

Figure 4: OSM safety criterion based on RN minimum distance at the evaluation times of Figure 3.

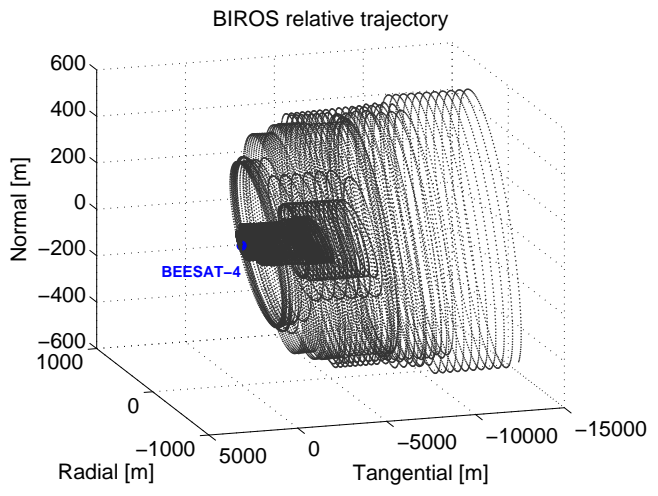

Figure 5: BIROS relative trajectory during the close-range commissioning phase in the BEESAT-4 orbital frame.

knows if a maneuver has been skipped. Meanwhile, passive safety allows each maneuver plan being interrupted prior to its completion without any harm.

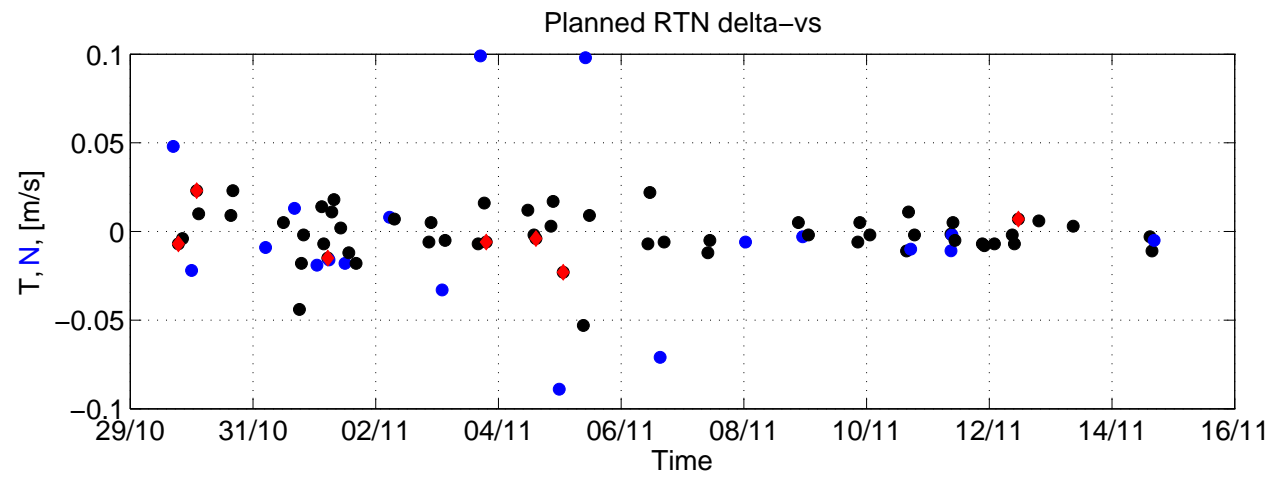

Figure 6: Commanded delta-vs during the close-range commissioning phase.

\section{CONSIDERATIONS ON TARGET VISIBILITY ASPECTS}

A first obvious consideration regarding the visibility of the target spacecraft is that pictures assume very different aspects depending on inter-satellite range a/o luminosity conditions (see some examples in Figure 7). At far-range the main difficulty lays in recognizing the target among all luminous spots in the image (e.g., faint stars, hot pixels, other satellites). Before the start of the experiment commissioning phase, it was even unknown to which distance the sensor would have been actually able to detect the tiny picosatellite. Radiometry analysis, in fact, provided spread results given the uncertainties on system, orientation, and sensitivity assumptions. At close-range, instead, the target identification becomes trivial but the orbit determination has to cope with the absence of stars in background a/o increasing centroid errors. All these considerations impact the navigation system and a dedicated discussion is carried out in References 10 and 11.

Another important aspect regards the need of a dedicated attitude mode to satisfy the visualtracking navigation needs. Such aspect becomes more demanding at closer inter-satellite ranges, to keep the target satellite in the field of view of the camera sensor, especially considering the spiraling 

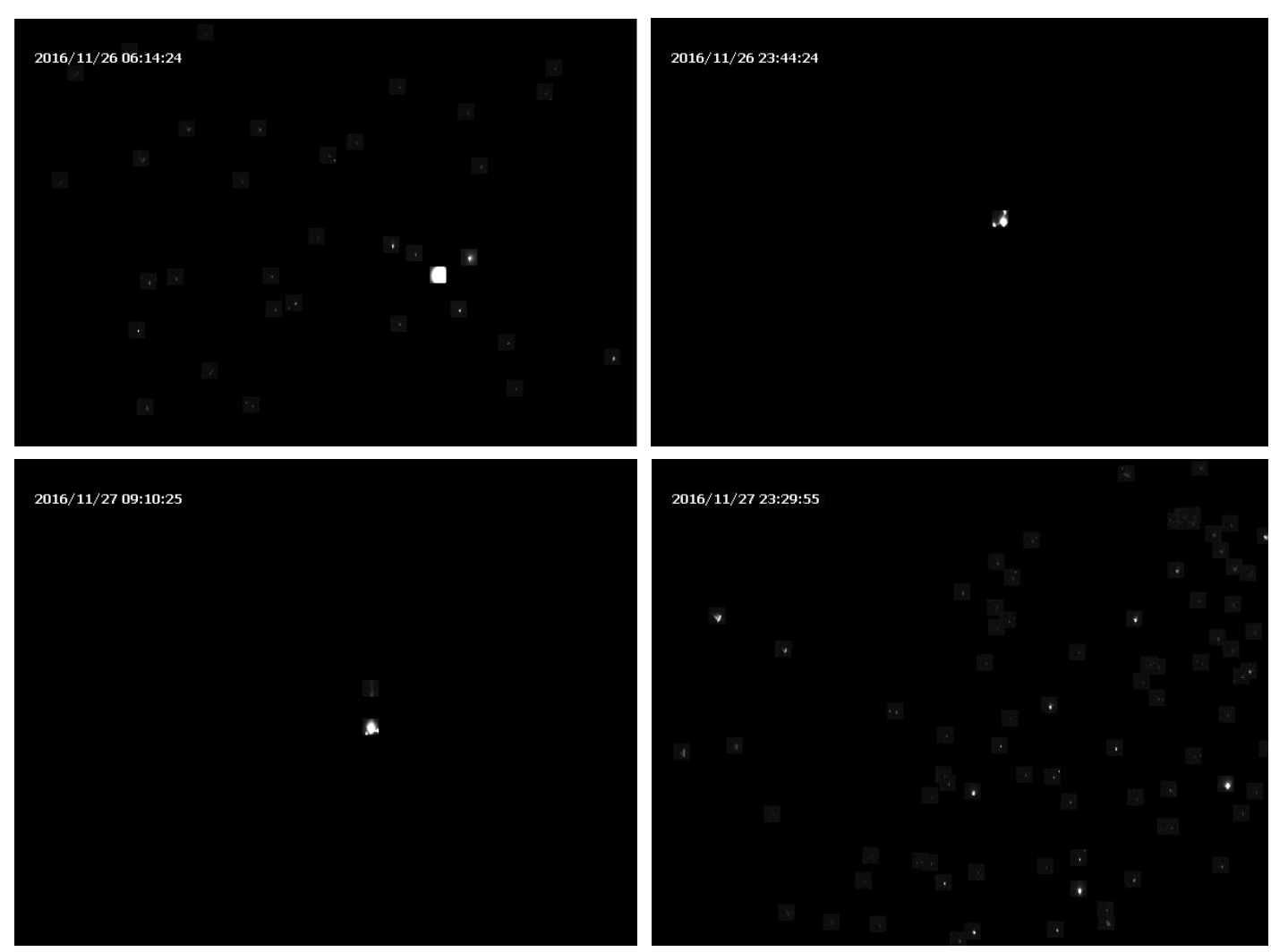

Figure 7: Some pictures taken at close-range during AVANTI.

approach of the AVANTI-concept. Table 1 summarizes how the so-called client observation attitude mode (COM) has been implemented. According to it, the boresight of the active camera head (i.e., $\mathbf{z}^{\mathrm{c}}$ where $c$ stands for camera-frame) is pointed to the local flight direction (i.e., BEESAT-4 is leading the formation during AVANTI) or tracks the line-of-sight to the target $\mathbf{u}^{\text {RTN }}$, with RTN (radialtangential-normal) denoting the local orbital frame. The remaining degree of freedom constituted by the rotation around the optical axis can be exploited to customize the attitude profile to the specific design of the chaser spacecraft. In the case of AVANTI, given the characteristics of BIROS, priority has been given to the improvement of the Sun-angle to solar panels, while guaranteeing a certain visibility angle of the GPS antenna to the Zenith (i.e., both GPS antennas are placed on the same side of the solar panels, that is normal to the $-\mathbf{z}^{\text {sat }}$ direction ${ }^{1}$ ). Particularly, the first option of Table 1 is to command the rotation angle $\alpha$ of the camera frame y-axis from the point where it is aligned to the projection of the local Zenith on the image plane. In this way a compromise between the two aforementioned preferences is achieved, during the whole orbit. The second method, instead, fosters the power budget aspect, seeking to minimize the angle of the Sun to the normal to panel during the portion of orbit in light. By contrast, while in eclipse, the satellite z-axis is directed to Nadir, to avoid pointing the GPS antennas to Earth. Thus, in this Sun-optimal profile, during every orbit BIROS rotates to re-orient its panel w.r.t. the Sun and performs two slews, entering and leaving the shadow region, while keeping the camera sensor towards the target s/c. Note that the COM definition of Table 1 generates a family of time-varying orientations obtainable by selecting: (i) the star-tracker camera head in use, (ii) first direction behavior, (iii) second direction preference (with additional tunable parameters). 
Table 1: Client Observation attitude Mode (COM) definition.

\begin{tabular}{|c|c|c|c|}
\hline First direction & Range & Second direction & Phase \\
\hline $\mathbf{z}^{\mathrm{c}} \|+\mathrm{T}$ & far & $\begin{array}{c}\mathbf{y}^{\mathrm{c}} \| \mathbf{R}_{\mathbf{z}^{\mathrm{c}}}(\alpha)\left(\left(\mathbf{I}-\left[\mathbf{z}^{\mathrm{c}} \times\right] /\left(\mathbf{z}^{\mathrm{c}} \cdot \mathbf{z}^{\mathrm{c}}\right)\right) \cdot \mp \mathbf{R}\right) \\
\text { or }\end{array}$ & always \\
\hline $\mathbf{z}^{\mathrm{c}} \| \mathbf{u}^{\mathrm{RTN}}$ & $\mathrm{mid} / \mathrm{close}$ & $\begin{array}{c}\min \angle\left(-\mathbf{z}^{\mathrm{sat}}, \mathbf{I}-\left[\mathbf{z}^{\mathrm{c}} \times\right] /\left(\mathbf{z}^{\mathrm{c}} \cdot \mathbf{s}^{\mathrm{RTN}}\right)\right) \\
\min \angle\left(-\mathbf{z}^{\mathrm{sat}}, \mathbf{I}-\left[\mathbf{z}^{\mathrm{c}} \times\right] /\left(\mathbf{z}^{\mathrm{c}} \cdot+\mathrm{R}\right)\right)\end{array}$ & $\begin{array}{l}\text { light } \\
\text { shadow }\end{array}$ \\
\hline
\end{tabular}

Figures 8 and 9 show how the COM Sun-optimal profile works, by plotting how Sun (in black during the phase of eclipse) and orbital frame directions (i.e., R, T, and Nadir) move in the BIROS body-frame sky-plot. For simplicity an axial-symmetrical baffle is considered, and a relative orbit presenting $\approx 1 \mathrm{~km}$ of mean along-track separation is simulated. Note that the isolated red points correspond to the attitude during eclipse.

In order to trade-off which camera head is better to use, one should consider the following aspects. First, given the absolute orbit of BIROS, the Sun is always blinding the active head during a portion of the orbit, even using so simplified geometrical considerations. Second, employing the camera head unit (CHU) labeled as 0 (left-view) implies having the remaining one (i.e. the attitude-only camera) continuously obstructed by the Earth. This fact degrades the accuracy achievable by the chaser attitude determination system, generating the following undesired issues. Maneuvers present larger execution errors disturbing the onboard navigation system which uses the commanded deltavs to improve the AO observability property. Moreover, at close-range, the noise of the observations remarkably increases, since the attitude information from BIROS has to be used to determine the orientation of the sensor, being no stars visible in the picture background. All the aforementioned considerations motivated the choice of using the unit- 1 as sensing instrument during AVANTI.

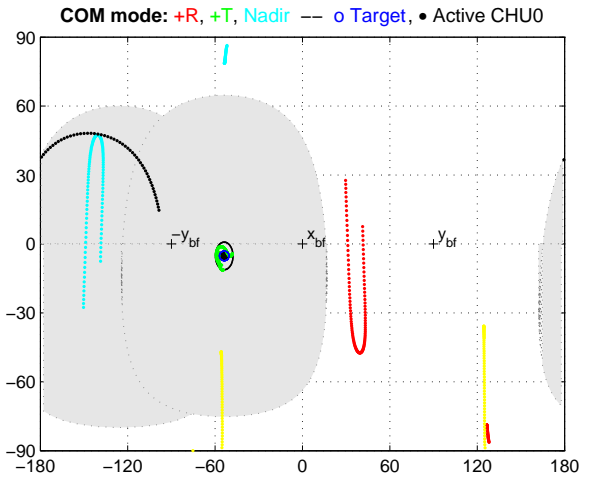

Figure 8: COM Sun-optimal using camera head 0.

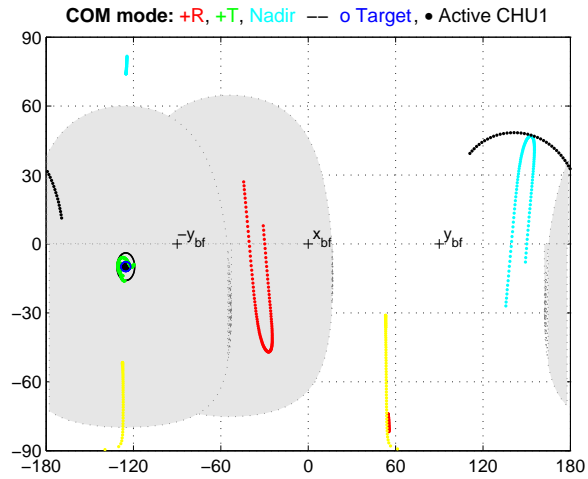

Figure 9: COM Sun-optimal using camera head 1.

As the more observations are collected the better it is to support AO navigation, BIROS had to spend plenty of time in COM mode. Thus it is important to consider which consequences this might bring to the functioning of the platform. For AVANTI the major problem revealed to be the thermal balance, since its thermal system has been sized to support the primary Earth-observation mission goal: all the time not dedicated to take pictures of hot-spots on the Earth surface is spent in an inertial-fixed Sun-pointing mode. Moreover, the radiator lays on the opposite side of the star-tracker. To understand the implications Figure 11 is helpful: line-of-sight and Sun tracks are 
plotted on the RTN unit sphere. The visual constraint of COM (i.e., directing a camera head mainly in flight direction) implies getting the Sun in the radiator once per orbit, disregarding which head is used. As a consequence, within AVANTI the additional cool-down attitude mode (CDM) has been implemented. ${ }^{1}$ This has the objective to dissipate as quickly as possible the heat, and it is entered following an onboard logic of temperature hysteresis loop monitoring some critical devices. Nevertheless, CDM generates a data gap in the observations, since the target does not fit in the camera field of view.

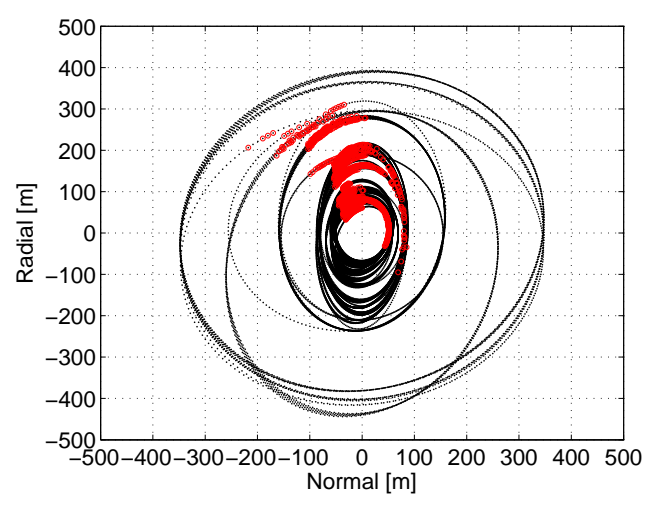

Figure 10: BEESAT-4 relative trajectory during the close-range commissioning phase.

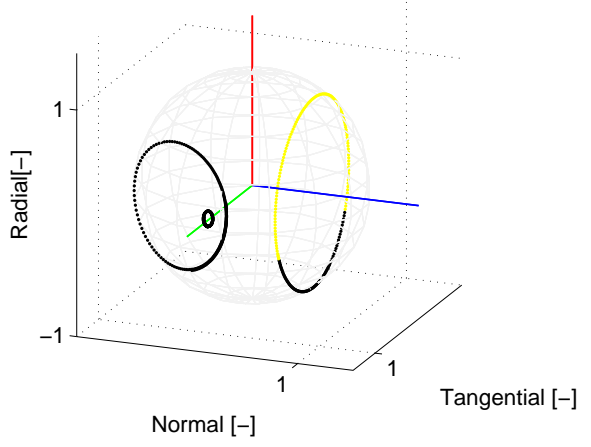

Figure 11: Target (mid- and close-range examples) and Sun tracks on the unit-sphere centered on the RTN frame.

Last considerations regard in which portion of the orbit observations are actually obtained, that is putting together attitude profile with the geometry of the relative motion. A spiraling trajectory presents a one-per-orbit oscillation in out-of-plane that varies the angular displacement between the boresight and the Sun direction, which describes a cone around the normal orbital axis. In-planewise, on the other hand, the relative eccentricity vector determines which part of the relative orbit is traveled during the phase of eclipse. Figure 10 shows the relative trajectory of BEESAT- 4 w.r.t. to BIROS projected on the local RN plane; red dots mark where observations are obtained. Within AVANTI, the preferred direction of the relative eccentricity vector has been chosen based on delta$\mathrm{v}$ budget and safety considerations, taking into account the effect of orbital perturbations during the time elapsed after the release of BEESAT-4. As a result, the point of minimum along-track distance (i.e., $\mathrm{R}=0$ and $\mathrm{N}<0$ in Figure 10) occurs during eclipse (i.e., the target cannot be imaged in the pictures). Note that, as the primary objective of AVANTI was to demonstrate AO approach in the far- to mid-range domain, getting observations more far way presented the benefit of an easier management of the field of view constraint. At close-range, the prevalent effect of the out-of-plane component improved the observation geometry so that in few occasions the measurements data arc lasted up to 35 minutes, clearly when no conflicting activities took place (e.g., maneuvers, CDM).

\section{DIFFICULTIES AT CLOSE-RANGE}

AVANTI demonstrated that the AO navigation approach can be used beyond the mid-range domain, to bring the chaser satellite at a separation distance where close-proximity specific sensors can be used. The main reason is that the navigation accuracy improves decreasing the inter-satellite distance, allowing to accept a larger noise of the measurements (e.g., centroid errors, downgraded knowledge of the orientation of the sensor). ${ }^{10,11}$ 
Aside from such peculiarities of the vision-based approach, at close-range difficulties arose due to the following technical constraints of the BIROS platform. First, the star-tracker is a far-range camera whose field of view corresponds to an area of solely $10 \times 15 \mathrm{~m}$ at $50 \mathrm{~m}$ of inter-satellite separation. Second, onboard computer and data handling of BIROS supported a maximum picture data-rate of 30 seconds. ${ }^{1}$ And, to achieve it, the only viable image compression format is the regionof-interest method (i.e., only a fixed-size pixel area around the luminous spots is kept). These characteristics are perfectly fine for running the AVANTI algorithms at far- to mid-range (which actually constituted the primary goal of the demonstration). At close-range, instead, these aspects demanded an attitude guidance able to keep the target in the picture, robustly against errors in the relative navigation solution. The design used in AVANTI, however, could not structurally achieve such robustness, since the COM attitude profile is generated propagating over the AVANTI time-step the current onboard navigation solution.

\section{DESIGN GUIDELINES FOR EXPLOITING THE AVANTI-CONCEPT}

This last section aims at summarizing constraints and degrees of freedom to exploit the AVANTIconcept design to future rendezvous missions. Still remaining in the framework of a minimalistic low-cost approach, few adaptations are suggested based on the experience collected so far.

The orbit scenario of an effective rendezvous mission is determined by the orbit of the target object (e.g., debris or client satellite). A vision-based AO approach can be exploited despite the orbit presents eclipses (i.e., AVANTI worked with 10 to 15 minutes of data arcs per orbit), provided a proper design of the navigation algorithms. On the other hand, an important aspect is to assess the relevance of non-conservative orbital perturbations (e.g., differential drag) acting in the scenario. If these are not negligible, in fact, the navigation system has to estimate them. In the case of differential aerodynamic drag, being its modeling greatly affected by the uncertainties of the unknown attitude and drag coefficient of the target spacecraft, a convenient option is to estimate the mean timederivative of the relative semi-major axis. This, in fact, catches the one-orbit mean value of such perturbing acceleration. ${ }^{15}$ Finally, relevant for the mission analysis study to achieve the far-range initial conditions, is the selection of rendezvous direction. Again the effect of orbital perturbations have to be considered. Within AVANTI, for example, for safety reason the element with larger ballistic coefficient (i.e., the target) lead the formation in flight direction, so that the natural effect of the differential aerodynamic drag made the satellite to drift apart form each other. ${ }^{6}$

Regarding the relative motion, the exploitation of passively safe relative orbits translate in the constraint of setting a specific phasing of the relative eccentricity and inclination vectors (i.e., 0 or 180 degrees). By combining this requirement with the delta-v consumption aspect, the most appealing option is to target $\delta e_{x}=\delta i_{x}=0$ (as performed in AVANTI). With this design, in fact, the perturbing effect of the secular perturbation due to $J_{2}$ on the out-of-plane motion is nullified for almost-bounded relative orbits (e.g., cheap control of inspection orbits). Therefore, the remaining degrees of freedom are the signs of y-components of the relative eccentricity and inclination vectors. Recalling the considerations of previous sections, these can be used to optimize the distribution of observations actually achievable across the orbit, given Sun-geometry and attenuation characteristics of the baffle of the optical sensor.

Regarding the chaser system design, care has to be paid on the number of camera heads and/or their location and mounting direction in the platform. A design exploiting three heads (like performed in PRISMA), gives the advantage to dedicate two heads exclusively for the attitude determination task. If not possible, the image-collection attitude mode has to be designed trading off the 
achievable attitude determination accuracy and the impact on other sub-systems of the chaser.

If a close-range phase is foreseen, some customized functionalities have to be also developed. In this domain, in fact, image sample rate and robust attitude guidance play an important role. Basically these generate constraints to the onboard data handling sub-system: the faster is the supported timestep the better it is for the navigation system. By exploiting the fact that at close-range the target identification becomes obvious, it would be advantageous (i.e., more robust) to connect the attitude control directly to the image processing output, with the objective to keep the target in the center of the field of view. Note that for inspection orbits, a specific attitude guidance might be needed to handle the portion of the relative orbit overshooting the target in flight direction.

\section{CONCLUSION}

AVANTI successfully demonstrated the feasibility of angles-only vision-based navigation to safely and autonomously rendezvous a noncooperative object in low Earth orbits. This paper critically presented advantages and limits of the guidance navigation and control system developed to support such flight demonstration. As a result, some design guidelines have been outlined to exploit such flight-proven concept to future rendezvous missions.

\section{REFERENCES}

[1] G. Gaias, J.-S. Ardaens, and C. Schultz, "The AVANTI experiment: flight results," Salzburg, Austria, $10^{\text {th }}$ International ESA Conference on Guidance, Navigation \& Control Systems, 2017.

[2] "The AVANTI weblog," http://www.dlr.de/rb/desktopdefault.aspx/ tabid-11685/\#gallery/28310.

[3] T. Weismuller and M. Leinz, "GN\&C Technology Demonstrated by the Orbital Express Autonomous Rendezvous and Capture Sensor System," No. Paper 06-016, American Astronautical Society, 2006.

[4] P. Bodin, R. Noteborn, R. Larsson, T. Karlsson, S. D’Amico, J.-S. Ardaens, M. Delpech, and J.-C. Berges, "PRISMA Formation Flying Demonstrator: Overview and Conclusions from the Nominal Mission," No. 12-072, Breckenridge, Colorado, USA, $35^{\text {th }}$ Annual AAS Guidance and Control Conference, 2012.

[5] "Technische Universität Berlin, BEESAT-4 website," https://www.raumfahrttechnik. tu-berlin.de/menue/forschung/aktuelle_projekte/beesat-4/parameter/ en/.

[6] M. Wermuth, G. Gaias, and S. D'Amico, "Safe Picosatellite Release from a Small Satellite Carrier," Journal of Spacecraft and Rockets, Vol. 52, No. 5, 2015, pp. 1338-1347. doi: 10.2514/1.A33036.

[7] M. Wermuth and G. Gaias, "Operational Concept of a Picosatellite Release from a LEO Satellite," Munich, Germany, 25 ${ }^{\text {th }}$ International Symposium on Space Flight Dynamics ISSFD, 2015.

[8] H. Reile, E. Lorenz, and T. Terzibaschian, "The FireBird Mission - A Scientific Mission for Earth Observation and Hot Spot Detection," Small Satellites for Earth Observation, Digest of the $9^{\text {th }}$ International Symposium of the International Academy of Astronautics, Berlin, Germany, Wissenschaft und Technik Verlag, 2013, pp. 184-196. ISBN 978-3-89685-574-9.

[9] S. Föckersperger, K. Lattner, C. Kaiser, S. Eckert, W. Bärwald, S. Ritzmann, P. Mühlbauer, M. Turk, and P. Willemsen, "The Modular German Microsatellite TET-1 for Technology On-Orbit Verification," Glasgow, UK, 59 ${ }^{\text {st }}$ International Astronautical Congress, 2008.

[10] J.-S. Ardaens and G. Gaias, "Angles-Only Relative Orbit Determination during the AVANTI experiment," Matsuyama, Japan, 26 "International Symposium on Space Flight Dynamics ISSFD, 2017.

[11] J.-S. Ardaens and G. Gaias, "Flight Demonstration of Spaceborne Real-Time Angles-Only Navigation to a Noncooperative Target in Low-Earth Orbit," University of Colorado Boulder, Colorado, USA, $9^{\text {th }}$ International Workshop on Satellite Constellations and Formation Flying, 2017.

[12] G. Gaias and J.-S. Ardaens, "Design challenges and safety concept for the AVANTI experiment," Acta Astronautica, Vol. 123, 2016, pp. 409-419. doi: 10.1016/j.actaastro.2015.12.034.

[13] G. Gaias, S. D'Amico, and J.-S. Ardaens, "Generalized Multi-Impulsive Maneuvers for Optimum Spacecraft Rendezvous in Near-Circular Orbit," Int. J. Space Science and Engineering, Vol. 3, No. 1, 2015, pp. 68-88. doi: 10.1504/IJSPACESE.2015.069361. 
[14] G. Gaias and S. D'Amico, "Impulsive Maneuvers for Formation Reconfiguration using Relative Orbital Elements," Journal of Guidance, Control, and Dynamics, Vol. 38, No. 6, 2015, pp. 1036-1049. doi: 10.2514/1.G000191.

[15] G. Gaias, J.-S. Ardaens, and O. Montenbruck, "Model of J2 Perturbed Satellite Relative Motion with Time-Varying Differential Drag," Celestial Mechanics and Dynamical Astronomy, Vol. 123, No. 4, 2015, pp. 411-433. doi: 10.1007/s10569-015-9643-2. 